\title{
Politique
}

\section{La bourgeoisie canadienne et les paramètres de l'alliance continentale}

\section{François Rocher}

Numéro 14, automne 1988

Sport et politique et Le NPD

URI : https://id.erudit.org/iderudit/040603ar

DOI : https://doi.org/10.7202/040603ar

Aller au sommaire du numéro

Éditeur(s)

Société québécoise de science politique

ISSN

0711-608X (imprimé)

1918-6584 (numérique)

Découvrir la revue

Citer cet article

Rocher, F. (1988). La bourgeoisie canadienne et les paramètres de l'alliance continentale. Politique, (14), 119-155. https://doi.org/10.7202/040603ar d'utilisation que vous pouvez consulter en ligne.

https://apropos.erudit.org/fr/usagers/politique-dutilisation/ 


\title{
LA BOURGEOISIE CANADIENNE ET LES PARAMĖTRES DE L'ALLIANCE CONTINENTALE
}

\author{
François Rocher \\ Université d'Ottawa
}

La question de la continentalisation de l'économie canadienne, particulièrement remise à l'ordre du jour dans le cadre du projet du gouvernement conservateur de libre-échange canado-américain, pose de nouvelles questions au cadre analytique de la «nouvelle économie politique». Ce dernier a mis l'accent sur les implications économiques, politiques et sociales des rapports de dépendance entre le Canada et les États-Unis dans une perspective analytique classiste. L'objet de notre démarche, qui se situe dans la tradition de la nouvelle économie politique canadienne, est d'appréhender la configuration des rapports sociaux, notamment ceux qui caractérisent les différentes composantes de la classe économiquement dominante, rapports sous-jacents à la politique commerciale continentaliste qui s'est développée au niveau central de l'État au cours des années 1970 et 1980 .

Notre démarche s'articulera en deux temps. D'abord nous chercherons à mettre en lumière les forces et certaines faiblesses du cadre général d'analyse et le schéma d'argumentation de la nouvelle économie politique telle que développée au Canada. Prenant appui sur les éléments du débat entourant le libre-échange 
canado-américain, nous tenterons, dans un deuxième temps, d'appréhender les dimensions politiques et sociales du rapport de dépendance. Il s'agira donc d'identifier les lieux d'expression des intérêts des différentes composantes de la bourgeoisie au sein de la société civile. En somme, nous croyons que la «domination externe» conditionnée par les intérêts américains passe par une intériorisation, sur les plans politique et économique, des conditions du développement économique continental. Ainsi, ce rapport de dépendance ne serait pas une réalité directement imposée de l'extérieur, mais s'expliquerait en grande partie par la dynamique interne de reproduction du capital selon un schéma de développement qui ne peut rompre avec les conditions de la dépendance.

L'objet de ce texte est de présenter les paramètres de l'alliance continentaliste et de montrer en quoi ils different des clivages traditionnellement identifiés. Bien que la politique commerciale libre-échangiste doit être analysée dans la mouvance de la restructuration de l'économie mondiale, nous n'aborderons que très marginalement cette question. Notre propos est plutôt de saisir comment s'articule le discours continentaliste au sein de la classe économiquement dominante au Canada, quels sont les intérêts en jeu et finalement en quoi cette option contribue à approfondir l'intégration continentale.

1. Démarche analytique de la "nouvelle économie politique»

Les travaux qui constituent ce qu'il est convenu d'appeler la «nouvelle économie politique» se sont principalement efforcés de mettre en lumière les incidences historiques, sociales et politiques de la dépendance de l'économie canadienne à l'égard des ÉtatsUnis (Drache et Clement, 1985). Ces recherches, en réaction à l'approche libérale, vont axer leurs analyses sur un certain nombre de questions dont notamment le procès de formation du capitalisme au Canada, l'inégalité des échanges entre le Canada et les États- 
Unis, la structure de propriété du capital, la configuration de la bourgeoisie canadienne eu égard à la prédominance des intérêts étrangers au Canada, le développement inégal des régions, le rôle spécifique que joue l'État aux niveaux central et régional à l'intérieur du processus de développement industriel et commercial, les effets de la dépendance au niveau de l'accumulation et de la circulation du capital, etc. Les contributions de l'approche de la «nouvelle économie politique» sont donc multiples et touchent à de nombreux champs. Nous allons, pour notre part, tenter de dégager certaines propositions concernant le développement économique, la configuration des rapports au sein de la bourgeoisie et, finalement, le rôle de l'État au Canada.

\section{Le développement économique}

Pour les théoriciens de la "nouvelle économie politique», il ne fait aucun doute que le Canada connaît une situation de dépendance équivalente, en termes de configuration économique, à celle que connaissent les pays latino-américains à l'égard des capitaux étrangers. La dépendance s'illustre particulièrement bien lorsque l'on considère les domaines du commerce extérieur, la provenance des investissements, la présence des firmes multinationales, l'emprunt du savoir-faire au niveau de la haute technologie, etc. De façon générale, la dépendance correspond à l'«invasion" de l'économie nationale par le capital étranger. Plus concrètement, ce processus se matérialise par la croissance des capitaux directs et par la présence de cadres supérieurs étrangers dans les secteurs les plus productifs de l'économie. Cette situation particulière est manifeste lorsque l'on compare la structure économique du Canada à celle des autres nations capitalistes du centre. Ainsi, pour Lumsden:

«What is significant about metropolitan capitalist countries, regardless of their population, is that their bourgeoisies have had sufficient autonomous strenght to counter balance the loss of part of their home market by themselves penetrating the most advanced markets. " $(1979,18,22)$ 
Une situation de «sous-développement» telle que le connaît le Canada ne correspond pas pour autant à l'absence de développement. Allant dans le même sens, Clement soutiendra que le développement dans un pays ne signifie pas nécessairement le développement du pays (1977A, 126). Ainsi, une formation sociale dépendante présente plusieurs niveaux de développement selon les secteurs économiques et les régions. La domination du capital étranger conduit à une situation de concurrence inégale où n'est laissée au capital local que la part non voulue par les investisseurs étrangers. Le capital autochtone n'a pas d'autre alternative que d'accepter son statut subalterne et d'orienter ses activités dans les secteurs marginaux ou ceux de la sous-traitance. L'ensemble du système est ainsi dominé par le capital étranger qui en détermine les grandes orientations.

Le caractère dépendant du Canada produit donc un double processus de développement inégal: d'une part, le Canada se trouve dans une position défavorable par rapport aux autres formations sociales capitalistes et, d'autre part, ce procès de développement est à la source d'inégalités entre les régions qui forment le Canada. Le continentalisme contribue à l'accentuation des disparités régionales dans la mesure où il perpétue les spécialisations régionales. De plus, il modifie la nature des rapports entre les paliers gouvernementaux au Canada du fait que la nouvelle orientation commerciale nord-sud et le système de succursales des firmes multinationales (branch-plant) peut contribuer à accroître le pouvoir politique des gouvernements provinciaux si ce mouvement n'est pas endigué par le gouvernement central. Ainsi, la fragmentation économique et politique du pays sert directement les intérêts des grandes entreprises multinationales (Stevenson, 1980, 16-28).

Par ailleurs, la présence des firmes multinationales, par l'exportation des capitaux qui en résulte et le contrôle exercé sur l'activité économique en général, illustre la subordination de 
l'économie nationale aux impératifs de croissance déterminés de l'extérieur. Cette subordination prend même le visage d'un nouveau colonialisme impossible à dépasser autrement que par une volonté politique de rapatrier les pouvoirs économiques. Cet énoncé constitue le fil conducteur adopté par les nombreux analystes pour qui ce néo-colonialisme est particulièrement perceptible à travers l'analyse du processus de pénétration de l'économie nationale par les investissements directs des entreprises multinationales. Ces dernières sont considérées comme les instruments par lesquels la domination économique des États-Unis s'exerce et se perpétue (Clement, 1977B, 71-84 et $1983,55-84)$.

La perspective analytique met donc en évidence le fait que cette dépendance économique à l'égard des États-Unis et la domination que ce centre exerce sur le Canada ne peuvent pas uniquement s'expliquer par des facteurs d'ordre démographiques ou géographiques tels la faible dimension du marché ou la proximité de la frontière étatsunienne. La différence déterminante entre ces deux économies n'en est pas une d'échelle mais bien de nature. Cette différence est particulièrement perceptible lorsque l'on considère les questions de la concentration des activités économiques et du poids relatif de chacune d'elle au sein de la structure commerciale et industrielle. S'ajoute à cette structure économique et industrielle tronquée le fait que la propriété des secteurs les plus rentables est entre les mains des intérêts étrangers. Cet ensemble de facteurs contribue à démontrer le caractère dépendant de l'économie canadienne et explique en grande partie le processus de «mal-développement» dans lequel elle est structurellement engagée.

L'utilisation de l'approche dépendantiste pour comprendre la réalité canadienne fait l'objet d'une remise en question depuis la fin des années 1970. Essentiellement, les critiques se rapportent à l'inadéquation entre les thèses dépendantistes et l'évolution récente du capitalisme au Canada. 
Les transformations de l'accumulation capitaliste au Canada et au sein du système mondial ont fait dire à plusieurs auteurs que le cadre d'analyse de la dépendance s'avère inadéquat pour comprendre les relations qui se sont établies récemment entre les États-Unis et le Canada ainsi qu'avec les autres nations qui forment le système économique international (Niosi, 1980; Resnick, 1982 et 1988; Caroll, 1986). Un certain nombre de facteurs sont énumérés pour fonder cette position: pensons entre autres aux phénomènes de la concentration des entreprises, à l'augmentation du contrôle autochtone sur les principaux secteurs économiques, au renforcement des banques canadiennes à l'étranger, à l'accroissement des interventions gouvernementales dans les secteurs productifs, au processus de multinationalisation de firmes canadiennes, à la réduction du contrôle étranger dans les secteurs manufacturier et énergétique et aux politiques visant à limiter les investissements étrangers au Canada (Éthier, 1985, 66; Drache et Kroker, 1983, 7-9). Ces nouveaux phénomènes sont à la source d'une reconsidération du caractère dépendant du Canada. Poussant plus loin, certains soutiennent même que le Canada doit maintenant être considéré comme un pays du centre.

Ces critiques nous semblent adopter une définition trop étroite de la notion de dépendance. Il est vrai de constater que le Canada a connu des modifications importantes aux niveaux de la propriété des entreprises et de l'exportation des capitaux. Le rééquilibrage entre le poids du capital canadien aux États-Unis et le poids du capital américain ici ainsi que la multinationalisation du capital canadien peuvent toutefois être interprétés comme des manifestations du déclin du Canada comme lieu d'investissements directs (Houle, 1987, 489-490). Ce déclin se traduit entre autres par la décroissance des dépenses de recherche et de développement (en \% du PNB), la détérioration de la balance commerciale de produits manufacturés de haute technologie, l'approfondissement de la dépendance commerciale envers les États-Unis et l'importance 
du commerce intra-société. De plus, il nous faut souligner que la dépendance s'exprime, pour une large part, par une capacité tronquée de définir ses orientations économiques et politiques au profit des impératifs productifs établis à l'extérieur. Il est entendu que la mondialisation du capital rend toutes les économies interdépendantes. Mais l'asymétrie des rapports économiques bilatéraux nous conduit à parler de dépendance pour décrire la situation du Canada à l'égard de son puissant voisin. Il n'y a donc pas nécessairement inadéquation entre le processus de maturation de l'économie canadienne et le maintien d'un rapport de dépendance dans la mesure où le premier ne peut se réaliser que dans le cadre d'un approfondissement du second. Il nous faut donc prendre en compte les facteurs externes qui permettent l'intériorisation des intérêts étrangers à travers des pratiques sociales déterminées. Ces seules critiques n'invalident nullement une démarche analytique qui se fonderait entre autres sur la notion de dépendance pour appréhender l'économie politique canadienne.

Toutefois, au-delà de cette simple nomenclature de phénomènes nouveaux qui obligent à reconsidérer le statut de dépendance du Canada, ce qui est mis en évidence est le fait que l'approche de la "nouvelle économie politique», en ne considérant que les seuls rapports Canada/États-Unis, néglige de prendre en compte la restructuration des rapports économiques et politiques induite par la nouvelle division internationale du travail (Éthier, 1983, 344).

Par ailleurs, l'attention portée aux facteurs économiques fait en sorte que ces derniers sont surévalués au détriment de la prise en compte des aspects sociaux qui conditionnent l'instauration du capitalisme au Canada. Ainsi, l'approche dépendantiste telle que développée au Canada met davantage l'accent sur l'articulation des facteurs externes qui conditionnent la dépendance plutôt que de mettre de l'avant une analyse qui tienne compte de la signification politique des processus économiques. Ces considérations mettent 
clairement en évidence la difficulté de la part de certains auteurs à saisir la dynamique sociale et politique sous-jacente aux rapports de dépendance qui caractérisent la formation sociale canadienne.

En somme, la dépendance est considérée comme une donnée «structurelle». De ce fait, l'accent est relativement peu mis sur les agents sociaux qui lui permettent de s'implanter et de se développer. L'empirisme, comme méthode de démonstration, domine l'approche dépendantiste au Canada. Il conduit à sousévaluer les forces sociales, forces perceptibles principalement par une analyse qualitative des positions politiques et sociales des divers agents et classes qui composent la formation sociale canadienne (Glenday, 1983, 353; Schmidt, 1981, 84).

Ainsi, nous devons exclure toute détermination a priori dans l'ordre d'explication entre les facteurs internes et externes. Il s'agit plutôt d'une combinaison d'éléments contradictoires qui procède tant de la «logique économique» et des rapports mondiaux de production que de la configuration interne des rapports de force. Les intérêts qui peuvent être identifiés sur la scène mondiale se doivent d'être appréhendés à partir de leur médiation avec les intérêts locaux, les classes autochtones et l'État au Canada. Pour ce faire, nous devons réévaluer l'articulation qui est généralement présentée chez les auteurs de la "nouvelle économie politique» au sujet des classes sociales et de l'État.

\section{La configuration de la bourgeoisie et ses rapports à l'État}

Si les aspects strictement économiques du continentalisme ont été largement développés, il n'en demeure pas moins que cette situation a eu aussi des répercussions importantes au niveau de la configuration des rapports sociaux et, plus spécifiquement, en ce qui concerne les contradictions que l'on retrouve au sein de la classe économiquement dominante. Cependant, les études qui ont été conduites dans la perspective de la dépendance du Canada 
à l'égard des États-Unis n'ont pas toutes appréhendé l'articulation des rapports au sein de la bourgeoisie canadienne dans les mêmes termes. Selon les auteurs, la place et le poids relatif de chacune des fractions de la bourgeoisie different sensiblement. La bourgeoisie est couramment divisée, dans la littérature socialiste canadienne, selon ses deux principales fractions: les fractions autochtone et compradore. Dans la foulée de la reconnaissance de la situation de dépendance, la fraction compradore de la bourgeoisie (regroupant généralement les agents qui sont au service des entreprises multinationales) est considérée comme dominante pour la période d'après-guerre et ce, tant aux niveaux politique qu'économique (Laxer, 1973, 35).

Ces considérations générales sont partagées par l'ensemble des auteurs qui font une analyse classiste de l'incidence de la dépendance sur la configuration de la classe économiquement dominante. Cependant, des divergences existent quant à la nature et à l'orientation des rapports qui se sont développés à l'intérieur de la bourgeoisie, notamment en ce qui concerne le rôle de la fraction compradore et du type d'alliance que cette dernière a conclu avec la fraction autochtone selon les secteurs d'activité.

Pour Wallace Clement, ces clivages hiérarchisés au sein de la bourgeoisie, fondés sur une division du travail, n'engendrent pas nécessairement des rapports contradictoires. Il semble plutôt se dégager une alliance conditionnée surtout par le fait que chacune des fractions de la bourgeoisie opère dans des champs d'activité relativement distincts et complémentaires (1977B, 73; 1975, 337). Cette relative harmonie entre les fractions dominantes de la bourgeoisie autochtone ne se reproduit cependant pas lorsqu'il s'agit de la fraction intermédiaire de la bourgeoisie nationale concentrée dans les activités manufacturières. Cette dernière entre directement en conflit avec les intérêts étrangers du fait que son champ de développement est le même (Clement, 1977B, 73). 
Pour sa part, Niosi soutiendra que Clement sous-estime le caractère conflictuel de l'alliance entre les deux fractions dominantes de la bourgeoisie au Canada. Il avancera la thèse voulant que:

\begin{abstract}
"The dominant strata of the Canadian capitalist class are so strong (not only in finance, commerce and transportation, but also in several manufacturing industries such as paper, agricultural machinery, distilleries, telecommunications equipement, metal refining, petrochemicals, etc.) that it cannot only challenge foreign competition in Canada, but also export to or invest in the U.S. in order to capture a large portion of the American market. The dominant strata of the Canadian bourgeoisie are not afraid of a continental economy. They have carved narrow but secure niches for themselves in many financial and non-financial industries and are quite able to compete in the North American market. This is not to say that the Canadian capitalist class is dissolving itself within a Continental bourgeoisie. In fact, some level of dependency and Continental integration is a profitable strategy for the dominant strata in Canada, even if it sacrifices many small and mediumsize local manufacturing firms." (1983, 133; voir aussi 1979, 121-2; 1980, 711)
\end{abstract}

Cette position vise à reconnaître la présence d'importantes firmes canadiennes dans d'autres secteurs que ceux traditionnellement identifiés par les nationalistes canadiens, à savoir la finance et le commerce. Ainsi, le déclin du contrôle étranger au cours des années 1970, particulièrement dans les secteurs des richesses naturelles, et le processus de transnationalisation de firmes canadiennes démontrent la présence d'une bourgeoisie autochtone montante relativement forte (Niosi, 1982). Cette reconnaissance ne signifie cependant pas que la bourgeoisie autochtone ait atteint son entière maturité. Pour Niosi, la consolidation de la bourgeoisie nationale ne modifie pas le caractère semi-industriel de l'économie canadienne ni la structure de dépendance qui occasionne toujours des distorsions importantes. Ce qui a changé au cours des années 1970 est la structure de propriété sans que cela affecte positivement, et de manière importante, la structure industrielle du Canada. La montée de firmes canadiennes dans des secteurs historiquement dominés par le capital étranger vient modifier l'appréhension des rapports entre les fractions de la bourgeoisie de deux façons: d'une part, il n'est pas possible d'affirmer que la bourgeoisie canadienne 
soit entièrement inféodée aux capitaux étrangers et, d'autre part, l'étude des rapports qui se nouent entre ces fractions est beaucoup plus complexe qu'il n'y paraît, du fait de la consolidation des intérêts nationaux dans des secteurs traditionnellement contrôlés par des intérêts étrangers.

Ces clivages analytiques concernant la configuration de la bourgeoisie canadienne se retrouvent aussi lorsqu'il s'agit d'appréhender la nature des rapports entre la bourgeoisie et l'État canadien.

La perception des rapports entre la bourgeoisie canadienne et l'État laisse place à des interprétations passablement divergentes, particulièrement en ce qui concerne l'identification de la fraction du capital dominant l'État à travers les multiples conjonctures. Le principal clivage pouvant être mis en évidence oppose ceux qui perçoivent l'État comme largement inféodé aux intérêts étrangers et ceux qui croient, au contraire, que les appareils gouvernementaux ont été investis par les intérêts de la bourgeoisie nationale.

Le premier courant a été notamment systématisé par Kari Levitt pour qui la dépendance croissante du Canada à l'égard de l'étranger conduit, sous forme d'une balkanisation politique, à l'absorption progressive du pays par le système américain. Le Canada connaît un processus d'homogénéisation de ses valeurs et de subordination de ses élites politiques et technocratiques (Levitt, 1973, 168-181). Par ailleurs, ce processus, fruit de la "colonisation" du Canada par la puissance impérialiste américaine, incite les détenteurs du pouvoir économique à s'associer de plus en plus aux intérêts étrangers et à approfondir le rapport de dépendance (Clement, 1977A, 299-301; Panitch, 1977, 99; Laxer 1973, 356). Ce processus de subordination ne s'explique donc pas essentiellement par la prise de contrôle de l'État par les agents de la fraction compradore de la bourgeoisie (Hutcheson, 1973, 174) mais plutôt par les orientations économiques et idéologiques, d'une part, de la vieille élite canadienne (particulièrement la 
fraction commerçante) qui favorise la stratégie continentaliste pour assurer son expansion et, d'autre part, de la bourgeoisie industrielle dans ses secteurs dominants (Layton, 1976, 151-2).

S'opposant en partie à cette vision, d'autres auteurs ont soutenu que l'État, loin de ne prendre en compte que les intérêts étrangers, a contribué à favoriser les capitaux autochtones par des politiques de canadianisation de l'économie et par la création de sociétés d'État permettant ainsi la croissance d'une bourgeoisie authentiquement nationale. Ainsi, pour Niosi, s'il est vrai que l'État a assuré l'expansion des investissements étrangers au cours de la période d'après-guerre jusqu'au début des années 1970, l'entrée en crise de l'économie américaine et les stratégies adoptées par le gouvernement Nixon (particulièrement la résurgence des barrières tarifaires) ont accentué le nationalisme économique d'éléments minoritaires de la bourgeoisie canadienne. Parallèlement à ce processus, des bourgeoisies régionales se sont formées remettant en cause la concentration de l'activité économique en Ontario. Ainsi, des stratégies politiques ont été mises en place ne favorisant plus uniquement la croissance des investissements étrangers, mais plutôt la consolidation d'éléments régionaux de la bourgeoisie, ceux-ci étant fortement appuyés par les pouvoirs politiques provinciaux (Stevenson, 1982, Fournier, 1978).

Ce type d'analyse s'oppose à celles réduisant quasi mécaniquement l'État canadien à un rôle de porte-parole des intérêts des firmes multinationales étrangères ou de l'administration américaine. Cette approche considère plutôt que les interventions de l'État, au cours de la dernière décennie, ont affaibli la fraction compradore de la bourgeoisie «en mettant la puissance économique et l'autorité juridique de l'État au service de la classe dominante locale» (Niosi, 1979, 121-2).

Bien que proposant des lectures différentes des alliances entre les fractions de la bourgeoisie et l'État, les deux courants que nous avons identifiés ne nous semblent pas antinomiques. 
L'angle de saisie des rapports contradictoires au sein de la classe économiquement dominante conduit à des considérations de nature différente. Alors que dans le premier cas était mis en lumière le fait que la croissance des éléments autochtones les plus importants de la bourgeoisie était intimement liée à l'intégration continentale, l'approche privilégiée par les seconds tend à mettre en évidence que l'État, tant aux niveaux fédéral que provincial, n'adopte pas des politiques allant à l'encontre des intérêts exprimés par la bourgeoisie autochtone. Or, il nous semble possible d'affirmer que l'accentuation du lien de dépendance ne produit pas que des effets négatifs sur le développement de la bourgeoisie nationale. Au contraire, ces rapports de dépendance prennent appui sur des fractions du capital canadien qui tirent des bénéfices de l'intégration continentale. La croissance d'une bourgeoisie autochtone ne correspond pas nécessairement au renforcement du pouvoir économique détenu par cette dernière dans la mesure où elle ne peut que très faiblement orienter son processus d'accumulation. Par ailleurs, l'État peut à la fois adopter des positions allant dans le sens des intérêts de cette section du capital tout en ne modifiant pas la nature des rapports économiques qui existent entre le Canada et les États-Unis.

Le parti pris économiste de la «nouvelle économie politique» va avoir une incidence directe sur la façon dont sont présentés et analysés les rapports de classes au Canada et leur articulation avec l'État. La métaphore du «néo-colonialisme» développée par Levitt, et les études qui s'en sont inspirées par la suite, a conduit à laisser de côté les rapports de classes qui se sont constitués au Canada et leur évolution ainsi que les facteurs proprement politiques qui ont balisé le procès d'accumulation du capital au Canada. Comme le mentionne à juste titre Panitch:

\footnotetext{
"While the neo-mercantilist approach ignores class relations, from a Marxist perspective an appreciation of class relations of exploitation and struggle is essential to make sense of what is going on in a society and in relations between societies.
} 
Without this, one is forced to see development or underdevelopment entirely as the creation of international relations or of ruling classes or of reified organizations. As a result, our ability to understand Canada as a society which is both rich and dependent is severely limired. 》 $(1981,9)$

Cette perspective, que nous partageons, permet de nous éclairer sur les rapports de classes qui sont inhérents à la dépendance comme phénomène global. Ainsi, les travaux dont nous avons fait état jusqu’à présent, bien que voulant tenir compte des intérêts de classes, ont présenté ces derniers dans une perspective généralement statique. Les politiques gouvernementales sont perçues comme répondant aux besoins exprimés par une fraction particulière de la bourgeoisie. Les clivages que nous avons identifiés concernant la configuration de la bourgeoisie et de ses rapports avec l'État portaient d'ailleurs sur l'identification de la fraction dominante et non sur la dynamique conflictuelle inhérente aux rapports de classes. Le mode général d'appréhension de l'État demeure donc largement insatisfaisant. Ce dernier est généralement perçu comme le représentant d'une fraction particulière de la bourgeoisie. Ce découpage est par ailleurs repris lorsque vient le temps de considérer les clivages entre les niveaux central et régionaux de l'État. Celuici est constamment saisi comme l'instrument d'une fraction de classe. Les alliances qui sont mises à jour ne modifient pas le caractère statique de l'identification des rapports entre l'État et la fraction dominante dans la mesure où elles sont le résultat d'un partage structurel des champs d'accumulation de la bourgeoisie. Ainsi, ces alliances ne sont pas le fruit d'un compromis politique entre les différentes forces en présence, mais correspondent à la mise en place d'une division relativement harmonieuse du travail.

De ce fait, la "nouvelle économie politique» ne réussit généralement pas à dépasser la conception instrumentaliste de l'État. Ce dernier se présente comme isolé des autres sphères d'activité et est défini a priori comme indépendant des forces sociales présentes au sein de la société canadienne. Pour notre part, nous croyons qu'il importe de tenir compte d'un ensemble 
plus complexe de facteurs. Il nous faut en effet, d'une part, comprendre le mouvement du capital au Canada eu égard à la situation de dépendance qui le caractérise et, d'autre part, identifier clairement les lieux d'expression des intérêts de classes au sein de la société civile et leur "transposition" au niveau des appareils de l'État.

\section{Rapports de classes et intégration continentale}

Nous avons préalablement soutenu que la dépendance se définit essentiellement comme un rapport économique conditionnant dont la mesure ne peut se limiter au contrôle exercé sur l'économie canadienne par des intérêts étrangers, et que cette dépendance s'inscrit dans une configuration particulière de rapports sociaux. L'intégration continentale, qu'elle soit privilégiée par les éléments compradore ou autochtone de la bourgeoisie, constitue un approfondissement de ce rapport de dépendance. Dans cette perspective, il importe de mettre en relief les orientations du monde patronal vis-à-vis un éventuel accord de libre-échange. Il y a longtemps que certains éléments de la bourgeoisie font la promotion de cette stratégie commerciale. Il importe de remonter un peu dans le temps et de considérer les motifs qui ont poussé les grandes associations patronales, depuis le début des années 1980, à réclamer une libéralisation des échanges ${ }^{\text {l. }}$.

1. Même si les différents organismes représentatifs ont pu faire état de leurs positions à de nombreuses reprises, nous avons particulièrement retenu celles exprimées dans le cadre d'études ou d'enquêtes publiques conduites par le gouvernement fédéral depuis la fin des années 1970, notamment par le Comité sénatorial permanent des affaires étrangères (dont les rapports ont été déposés en 1978 et 1982), le Comité mixte spécial du Sénat et de la Chambre des communes sur les affaires extérieures (dont les audiences publiques se sont principalement tenues au cours de l'été 1985) et la Commission royale sur l'union économique et les perspectives de développement du Canada communément appelée la Commission Macdonald. Chacun de ses comités avait entre autres pour mandat d'examiner les relations économiques canado-américaines. $\dot{A}$ ces occasions, les différents organismes retenus ont fait valoir leur point de vue sur cette question tout en exposant les problèmes auxquels les différentes branches manufacturières ou l'économie dans son 
Nous cherchons aussi à briser l'impression unanimiste qui se dégage des prises de position de la bourgeoisie canadienne. Il nous semble erroné de prétendre que tout le milieu des affaires est d'emblée favorable au libre-échange. Les clivages qui se présentent méritent qu'on les examine quelque peu. Cette analyse peut nous permettre de jeter un éclairage nouveau sur les contributions traditionnelles de l'économie politique canadienne concernant la question de l'intégration continentale.

Jusqu'à présent, les rapports entre la situation de dépendance et la classe dominante ont principalement été déterminés dans le cadre du débat portant sur les politiques visant à limiter les investissements étrangers ou encore à soutenir certains éléments autochtones de la bourgeoisie à travers des programmes de subventions ou de canadianisation de l'économie. Schématiquement, l'interprétation traditionnelle sourenait que la fraction compradore de la bourgeoisie, tout comme les fractions financière et commerciale de la bourgeoisie autochtone, étaient favorables à l'accroissement des investissements étrangers et participaient, de ce fait, à l'approfondissement de la dépendance du Canada à l'égard de l'économie continentale. D'une manière moins tranchée, les éléments dominants de la bourgeoisie manufacturière canadienne ayant atteint une maturité leur permettant de concurrencer les producteurs américains, tout en voulant conserver les aspects symboliques du programme de tamisage des investissements étrangers, étaient favorables à l'élimination des restrictions imposées à la mobilité des capitaux

ensemble devaient faire face. L'analyse des documents publics, bien qu'éclairant à bien des égards, n'est pas sans causer plusieurs problèmes. Comme le mentionne I. A. Litvack, qui procède à une analyse fondée sur des données similaires: "It is apparent from an examination of the submissions made by the associations that there are problems of definition, absence of adequate date, and lack of knowledge of the U.S. political system and trade legislation. (...) As a general observation, many of the associations do not have a clear understanding of what constitutes a free trade agreement, more specifically the general economic, political and legal parameters of such an arrangement. (...) Consequently, certain of the submissions lack focus, don't come to grips with the key issues and therefore have little to offer in the way of concrete and realistic suggestions». (1986, 30-31). 
entre le Canada et les États-Unis. Ainsi, la fraction dépendante de la classe dominante était, dans l'ensemble, bien disposée face aux investissements américains. Par ailleurs, une orientation plus restrictive, que plusieurs ont qualifiée de nationaliste, émergeait des points de vue exprimés par la fraction non monopoliste de la bourgeoisie autochtone.

Ces clivages au sein de la bourgeoisie, quant à l'ouverture à afficher concernant les investissements étrangers, sont donc déterminés, d'une part, par la structure de propriété et, d'autre part, par l'envergure des activités des différentes entreprises. S'inspirant de cette approche, certains auteurs ont abordé le débat sur l'adoption d'une politique commerciale d'orientarion continentaliste en soutenant que les éléments de la fraction compradore de la bourgeoisie, peu importe les branches dans lesquelles ils sont implantés, seraient favorables à une libéralisation des échanges canado-américains; la bourgeoisie autochtone devrait afficher une position plus éclatée dans la mesure où les entreprises à caractère monopoliste ainsi que les grandes institutions financières seraient généralement favorables à une telle option alors que les entreprises non-monopolistes favoriseraient soit le statu quo soit une version édulcorée de libéralisation permettant de conserver des mesures protectionnistes ou de soutiens publics sectoriels (Brunelle et Deblock, 1987, 39). Or, le tour d'horizon des positions exprimées au sein de la bourgeoisie que nous avons effectué ne nous permet pas de cautionner cette hypothèse.

Éléments de la rhétorique libre-échangiste de la bourgeoisie

Les grandes associations de la bourgeoisie canadienne se sont faites les avocates du libre-échange canado-américain ${ }^{2}$. Pour plu-

2. Notamment la Chambre de commerce du Canada, le Conseil d'entreprises pour les questions d'intérêt national (mieux connu sous le nom de Business Council on National Issues), la Fédération canadienne de l'entreprise indépendante, le Conseil canadien 
sieurs, elles sont même à l'origine de cette politique commerciale (Langille, 1987, 64-70; 1988, 102-7; Cameron, 1986, xiv-xvii). Nous pouvons rapidement invoquer les arguments avancés par les éléments de la bourgeoisie qui soutiennent la libéralisation du commerce bilatéral.

Premièrement pour l'Association des manufacturiers canadiens, le problème le plus important auquel est confrontée l'économie canadienne s'avère la faible dimension de son marché. La compétitivité des entreprises canadiennes serait donc limitée par des facteurs d'ordre interne dont le plus important serait l'étroitesse du marché intérieur. Cette réalité empêcherait de maximiser les avantages de l'implantation de séries de production à plus grande échelle. Malgré de nombreux programmes destinés à encourager les exportations, la situation du Canada n'a cessé de se détériorer, notamment en ce qui concerne l'exportation des biens manufacturés (Canadian Manufacturers' Association, 1983, 3-4).

Un second argument présenté est à l'effet que le Canada doit faire face à une transformation importante des conditions dans lesquelles les échanges internationaux se réalisent. Ces conditions font en sorte que le Canada ne présente plus les mêmes avantages comparatifs qu'auparavant. La transformation observée s'expliquerait entre autres par la mise en place d'un nouvel environnement concurrentiel sur la scène mondiale. Celui-ci s'est implanté sous l'influence des développements technologiques rapides et de la spécialisation du commerce international. Dans ce processus, sont ainsi apparus de nouveaux concurrents qui posent un défi de taille pour l'économie canadienne, notamment en ce qui concerne la nécessité de suivre la progression des innovations technologiques, et qui appellent plus que jamais à l'adaptation et à la spécialisation (Crookel, 1984, 26-31).

des petites entreprises, le Conseil canadien du commerce de détail, l'Association des manufacturiers canadiens, l'Association canadienne d'exportation, l'Association des importateurs canadiens. 
Cet ensemble de facteurs fait peser une lourde menace sur la place du Canada au sein de l'économie internationale. Pour l'Association des importateurs canadiens, la diminution de la demande des biens et des ressources produits au Canada serait attribuable à l'augmentation relative des prix de ces produits (Canada, 1985, fascicule 5, 21). Une telle situation découlerait d'une baisse de la productivité, d'une croissance des coûts des facteurs de production et des lois et règlements imposés par les différents gouvernements au Canada. La conclusion d'un accord commercial global est présentée comme une condition nécessaire pour assurer le renouveau économique et accrôitre la compétitivité du Canada au plan international.

Troisièmement la croissance de l'interdépendance exige que le Canada devienne un acteur plus actif au sein des organismes internationaux, le GATT en étant un exemple. Mais compte tenu des incertitudes entourant une possible réouverture des négociations multilatérales, de la montée rapide du protectionnisme non seulement en Europe, mais aussi aux États-Unis, du fait qu'il faudrait attendre de cinq à dix ans après le début des négociations avant que des résultats perceptibles d'une nouvelle conférence du GATT n'apparaissent pour le Canada, le bilatéralisme est largement préféré au multilatéralisme ${ }^{3}$.

Le dernier argument, qui découle directement du précédent, est probablement le plus important. La problématique bilatérale est privilégiée en réponse au protectionnisme qui se développe aux États-Unis. Ainsi, le Canada a besoin d'une stratégie destinée à contrer les mesures restrictives pouvant être adoptées par le

3. Comme le soulignait J. A. Finlayson, directeur de la recherche au BCNI, "Canada industry has become more dependent on the United States as an export market. (...) Past effort to diversify Canadian Trade by increasing exports to other countries have not been notably successful. There is little reason to expect that significant new markets will soon open up to Canadian manufactured and high value-added exports. For many Canadian companies, this puts a premium on securing and, if possible, improving the access which they now enjoy to the United States", (1985, 30) 30. 
gouvernement américain (Association des manufacturiers canadiens, 1984, 91-2).

Pour l'AMC, ce sont les multiples barrières non tarifaires qui inquiètent le patronat canadien. Ces barrières dressent de sérieux obstacles aux entreprises canadiennes qui cherchent à écouler leur production sur le marché américain ${ }^{4}$. Un sondage, réalisé par l'AMC à la fin des années 1970 auprès de plus de 1,000 entreprises canadiennes, s'est intéressé entre autres à la façon dont ces dernières percevaient les lois du gouvernement américain concernant l'approvisionnement (Canada, 1980, fascicule 14, 54). Pour les entreprises sous contrôle canadien, à peu près $20 \%$ ont déclaré que les règlements américains avaient des conséquences graves ou modérées sur leur potentiel de croissance.

Les motifs habituellement énumérés par les éléments hégémoniques de la bourgeoisie ne fondent pas à eux seuls le soutien à la stratégie fédérale de libéralisation des échanges. D'autres enjeux méritent d'être soulignés, notamment en ce qui concerne le rôle de l'État au Canada, les investissements étrangers et la réalisation de l'union économique au Canada.

La question du libre-échange conduit à soulever l'épineuse question de la souveraineté de chaque pays dans la poursuite de leurs politiques. Le traitement de ce problème, par le patronat, se fait sur une base essentiellement pragmatique. Pour la Chambre de commerce du Canada, il s'agit même d'un faux débat (Canada, 1985, fascicule 5, 23). On ne peut opposer les notions d'amélioration du commerce et de souveraineté. Le raisonnement est simple: toute restriction au commerce se traduit inévitablement par un abaissement du niveau de vie. Dans ces circonstances, le pays qui adopte une telle politique grève son développement économique et culturel; à terme, c'est son autonomie et son indépendance qui

4. C'est aussi la position de I'Association canadienne d'exportation, (Canada, 1985, fascicule 4, 34) ainsi que de l'International Business Council of Canada (1983, 2). 
sont remises en question puisqu'il ne dispose plus des ressources financières suffisantes pour soutenir de nouveaux programmes étatiques. De ce fait, la meilleure façon de préserver la souveraineté du Canada est d'adopter toute politique pouvant assurer la croissance économique. Cet argument se fonde sur la conviction que l'accord pourrait favoriser le développement économique. Qui plus est, il implique que la richesse ainsi créée serait équitablement redistribuée entre les individus et les différentes régions du pays.

Cependant, au-delà de l'équation libre-échange $=$ croissance $=$ richesse $=$ accroissement de la capacité d'intervention étatique, certains reconnaissent que cette politique commerciale risque de modifier profondément les rapports existants entre, d'une part, l'État et la société et, d'autre part, les grandes corporations et les autres intervenants dans l'économie. Force est d'admettre qu'à l'intérieur de ce processus, la capacité d'intervention des gouvernements s'en trouverait réduites. Il apparaît d'évidence que toute initiative canadienne en matière de politique commerciale doit se faire sur la base d'une négociation où les deux parties acceptent de faire des concessions. On ne peut faire abstraction des coûts économiques et politiques d'une telle orientation. Même pour les représentants de l'AMC, une harmonisation des politiques doit être envisagée. Cette dernière implique entre autres que le

5. La position énoncée par l'AMC est on ne peut plus claire à ce sujet: "Au fur et à mesure que nous allons lever les obstacles aux échanges, pour laisser entrer et sortir librement les marchandises, il est évident que nous allons réduire le niveau d'indépendance politique au Canada. Il n'y a pas de quoi s'alarmer. Tout simplement, lorsque nous demanderons à nos industries de concurrencer l'industrie américaine, lorsque les restrictions aux échanges auront été abolies, nous au Canada allons être obligés de créer les mêmes conditions au Canada que celles qui existent aux États-Unis, que ce soit le régime d'assurance-chômage, l'assurance-sociale, le coût du gouvernement, le niveau d'imposition, etc. En fait, tout l'environnement socioéconomique devrait pouvoir se comparer avec ce qui existe aux Étars-Unis pour donner à l'industrie canadienne la chance raisonnable de produire à des coûts compétitifs, et cela veut dire que nous aurons moins de liberté pour créer, au Canada, un environnement très différent de celui qui existe aux États-Unis. Dans la réalité, je pense que c'est de cela qu'il faut parler quand on parle de libre-échange. " (Canada, 1980, 41). 
Canada établisse un niveau d'imposition des entreprises qui soit compétitif. D'autre part, il semble que certaines branches manufacturières vont rencontrer des problèmes d'ajustement (Association des manufacturiers canadiens, 1984, 94). Il faut cependant noter qu'aucune association n'a fait une analyse d'impact pour chacun des secteurs industriels. Mais il est certain, selon elles, qu'une libéralisation favoriserait les entreprises de fabrication qui sont d'envergure internationale. Une telle orientation aurait un impact certain sur le processus déjà bien amorcé de concentration industrielle favorisant surtout les grandes entreprises.

Ce discours sur la souveraineté est accompagné d'une profession de foi sans équivoque en la valeur de la régulation naturelle opérée par les forces du marché (Canada, 1980, fascicule 5, 24). L'ajustement et l'adaptation sont des facteurs récurrents de la vie économique. Conséquemment, il ne faut pas, selon la Chambre de commerce du Canada, accorder à ceux qui refusent de s'adapter une part du marché garantie à vie. Une telle pratique constitue plutôt une discrimination injuste qui gêne l'efficience du transfert des ressources vers les secteurs en croissance.

De manière générale, les politiques protectionnistes sont dénoncées comme étant inéquitables envers les entreprises qui sont les plus productives parce que mieux adaptées aux nouvelles conditions du commerce international. En somme, même si ces politiques sont parfois efficaces, elles doivent être évitées puisqu'elles retardent la nécessaire adaptation. La protection accordée à certaines branches industrielles au Canada ne vise d'ailleurs pas à limiter la concurrence provenant des États-Unis.

L'intégration continentale devrait donc se traduire par une diminution du rôle de l'État dans la gestion des rapports économiques. Les gouvernements devraient adopter des politiques compatibles avec celles des États-Unis, au nom de l'efficacité économique. Poussant plus loin la réflexion, il apparaît clairement que l'instauration du libre-échange constitue un pas dans la direction 
de la réduction de la capacité de l'État à définir une politique économique ${ }^{6}$. D'autre part, les programmes de soutien aux entreprises en difficulté devraient être révisés, ainsi que les politiques de soutien régional.

Ce processus d'intégration implique une modification de l'attitude du Canada à l'égard des investissements étrangers. L'établissement de relations bilatérales plus étroites implique que le Canada doive éviter d'adopter des politiques ayant pour résultat d'exacerber les tensions canado-américaines (Association des manufacturiers canadiens, 1984, 30; Canadian Export Association, 1983, 3). Il faut reconnaître que les investissements étrangers ont et continueront à contribuer de façon significative au développement économique national. De plus, l'accès accru au marché américain devrait conduire à l'attribution de mandats mondiaux de production pour les filiales de sociétés étrangères (Association des manufacturiers canadiens, 1984, 35).

D'autre part, la question qui demeure est évidemment la suivante: en l'absence de tels mandats mondiaux de production, les entreprises multinationales risquent-elles de relocaliser leur production aux États-Unis? Pour le patronat, et notamment le Conseil d'entreprises pour les questions d'intérêt national, ce danger n'est pas réel (Canada, 1985, fascicule 17, 46). Au contraire, dans une conjoncture où les obstacles tarifaires sont de moins en moins élevés, ces firmes pourraient tout aussi bien relocaliser leurs activités au sud de la frontière canadienne sans qu'il y ait un

6. Commentant les termes de l'accord préliminaire conclu en octobre 1987, l'AMC dira: «il doit être mentionné que le Canada (tour comme les É-U.) s'est engagé à ne pas créer d'avantage ni à faire de discrimination en sa faveur. Il y a un engagement mutuel de leur part à jouer les règles du jeu et à procéder à des consultations, ce qui en retour mettra l'accent sur une concurrence réelle et sur de vrais avantages comparatifs. Ces contraintes sont-elles acceptables? Du point de vue de la philosophie de l'AMC, la réponse est oui, puisque cet accord nous dirige vers une économie basée sur le marché, dépolitise les décisions économiques et élimine les interventions généralement considérées improductives et discriminatoires». (Association des manufacturiers canadiens, 1987, 14), les soulignés sont de nous. 
accord de libre-échange. Poussant plus loin le raisonnement, cette association soutient que l'absence d'un tel accord pourrait au contraire inciter certaines entreprises canadiennes à transférer leurs installations aux États-Unis pour éviter les politiques protectionnistes qui peuvent être imposées par le gouvernement américain.

Finalement, mentionnons que la capacité concurrentielle des firmes canadiennes dépendra aussi de facteurs qui ne sont pas tous liés à l'intégration continentale; en plus du taux de change, il faut aussi considérer, selon l'AMC, les politiques gouvernementales susceptibles de placer l'industrie canadienne dans une position désavantageuse par rapport aux États-Unis. L'Association des manufacturiers ainsi que la Chambre de commerce mentionnent, à titre d'exemples, l'existence de certaines politiques agricoles, la promotion par les gouvernements des échanges, le financement à l'exportation destinée aux entreprises régionales de toutes dimensions et certaines restrictions commerciales imposées par les provinces qui nuisent tant au commerce international qu'au mouvement des capitaux à l'intérieur même du Canada (Canada, 1985, fascicule 4, 8; fascicule 5, 21). Ces mesures font en sorte que le Canada connaît un marché moins bien intégré que peut l'être celui de la CEE. De ce fait, l'intégration continentale, dans l'esprit du patronat, doit être accompagnée d'une amélioration de l'union économique canadienne.

Les considérations régionales qui ont marqué la conduite de la politique canadienne doivent être remplacées par une vision plus nationale de la réalité économique. En d'autres termes, les politiques de développement régional ne doivent pas nuire à la consolidation de l'union économique. La grande bourgeoisie fait valoir que la compétitivité internationale exige la formation d'une masse critique d'industries spécialisées dans une région et non pas dix. La concentration industrielle est donc préférable à l'éparpillement. 
En somme, les porte-parole autorisés de la grande bourgeoisie préconisent une plus grande intégration continentale. Cette dernière vise des objectifs à court et à long terme. À court terme, la grande bourgeoisie cherche à contrer les menaces protectionnistes qui se font jour aux États-Unis. Elle désire avant tout assurer une stabilité dans ses échanges bilatéraux et circonscrire le plus possible les barrières qui peuvent être érigées et qui menaceraient les flux commerciaux. Il ne faut pas oublier que le Canada est le partenaire commercial le plus important des Américains; il doit faire face au mécontentement grandissant aux États-Unis et qui prend sa source dans un déséquilibre commercial grandissant. Au-delà de cet objectif à court terme, la libéralisation des échanges est perçue comme une option possible pour améliorer la compétitivité internationale de l'industrie canadienne. Même si théoriquement tous bénéficieraient d'une telle intégration, il apparaît clairement que ce sont les grandes entreprises exportatrices qui comptent tirer le plus profit d'un accès élargi au marché américain. Une telle orientation ne peut à elle seule résoudre tous les problèmes que rencontre l'économie canadienne, mais elle contribuerait certainement, aux dires de la grande bourgeoisie, à accélérer les mouvements de rationalisation et de spécialisation industrielles.

\section{L'alliance continentaliste: une nouvelle perspective}

Il appert que la très grande majorité des plus importantes associations regroupant les éléments dominants de la bourgeoisie canadienne s'est prononcée en faveur de la libéralisation des échanges commerciaux. Par ailleurs, ce large soutien de la part de la grande bourgeoisie ne traduit pas nécessairement une identité de point de vue de la part des différentes fractions de la classe économiquement dominante. Deux aspects méritent ici d'être soulignés. D'abord, le soutien au libre-échange repose sur des motifs différenciés. Il reflète soit des stratégies d'investissements différentes, soit des 
motifs d'ordre idéologiques d'inspiration néo-conservatrice. Ensuite, bien que les positions publiques de ces associations peuvent laisser croire au consensus, une analyse plus pointue nous démontre un clivage important au sein de la bourgeoisie entre les éléments qui sont actuellement orientés vers le marché américain et les autres qui desservent presque uniquement le marché intérieur.

Mentionnons que la multinationalisation de certaines firmes canadiennes a certainement contribué à modifier l'attitude de bon nombre d'entreprises autochtones tant à l'égard du marché américain que des politiques économiques pouvant être définis par le gouvernement canadien. Ainsi, le processus d'internationalisation du capital canadien s'est traduit, d'une part, par une croissance des investissements en sol américain et, d'autre part, impose une restructuration industrielle dans le but de réduire significativement les coûts de production. Rappelons à ce sujet une enquête menée en 1985 par l'Institut C. D. Howe auprès d'entreprises canadiennes ayant des filiales implantées aux États-Unis (Ministères des Affaires extérieures, 1986?, 11). Il ressortait de cette analyse que pour la très grande majorité des répondants, la décision d'investir aux États-Unis découlait de la volonté de bénéficier des avantages procurés par l'accès à un large marché et de la proximité de la clientèle. Par ailleurs, pour plus du tiers des entreprises manufacturières canadiennes ayant investi aux États-Unis, cette décision était en partie justifiée par la nécessité de contourner les multiples barrières tarifaires et non tarifaires. La libéralisation du commerce canado-américain doit donc être comprise comme un moyen d'accroître l'accès à un marché important pour un bon nombre d'entreprises multinationales canadiennes qui doivent poursuivre leur processus de concentration et de continentalisation (Langille, 1987 , 72; Kierans, 1987, 6-9; Resnick, 1988, 16). Cependant, toutes les multinationales canadiennes implantées aux États-Unis ne poursuivent pas les mêmes objectifs: 
" in the case of most companies in faster growing industries, to extend their sales into markets which otherwise would be closed to them, or in which they could not otherwise compete effectively. For this group, overcoming trade barriers, and serving the domestic market were the governing reasons for investment abroad.

In the case of most companies in slower growing industries, to modify their production capacity, their product lines or even their business. For this group, strengthening the existing business of the Canadian parent and diversifying into other business were the governing reasons." (Ministère des Affaires extérieures, 1986?, $12)^{7}$.

L'option commerciale continentaliste répond donc aux intérêts des multinationales canadiennes implantées aux États-Unis. Elle s'inscrit dans un mouvement de pénétration du marché américain déjà bien amorcé et en faciliterait la poursuite. Ce processus traduit un nouvel «esprit de confiance» des entrepreneurs canadiens s'inscrivant dans la «maturation» du capitalisme autochtone au Canada, reflétant une amélioration de la position de la bourgeoisie nationale face aux capitaux étrangers. Toutefois, le libre-échange ne peut être interprété comme manifestant une diminution de la dépendance du Canada à l'égard des États-Unis. Bien que nous reconnaissions les développements récents au sein du mode d'accumulation au Canada, force est d'admettre que la continentalisation va contribuer à lier davantage les décisions d'investissements prises au Canada aux conditions de développement économique imposées par nos voisins. Comme le mentionne à juste titre Kierans, «these negociations have one object, to smooth the path of Canadian corporate wealth into American markets and citizenship. What better way to avoid American contervail and anti-dumping laws than to become American nationals?» $(1987,9)$.

Certaines études ont aussi tenté de démontrer que le libreéchange répondait aux intérêts des entreprises américaines implantées

7. Les soulignés ne sont pas de nous. Le minisrère reprenait ici les principales conclusions d'une enquête conduite par l'International Business Council of Canada en 1985 auprès de dix-huit firmes multinationales canadiennes implantées aux États-Unis. 
au Canada (Rugman, 1987, 85-90) ${ }^{8}$. Cependant, ces dernières n'investissent pas au Canada pour les mêmes raisons que les entreprises canadiennes le font aux États-Unis. Une enquête réalisée par le Ministère des Affaires extérieures (1986?, 8) auprès des maisons mères démontrait essentiellement deux choses ${ }^{9}$. D'abord, que les barrières tarifaires et non tarifaires imposées par le Canada n'avaient que peu d'impact sur les décisions d'investissement au Canada. D'autre part, que seulement le cinquième des firmes consultées avait indiqué que le libre-échange aurait un impact positif sur leurs décisions futures d'investissement, bien que le quart ait signalé qu'une telle orientation se traduirait par une spécialisation des activités productives. Cette enquête indiquerait que les coûts d'ajustement seraient somme toute mineurs pour les filiales américaines, reflètant le faible impact des barrières tarifaires au niveau des décisions d'investissement et l'importance des échanges intra-firmes.

En somme, l'accord de principe des multinationales américaines ayant des activités au Canada repose sur des motifs fort différents de ceux des entreprises canadiennes faisant affaire aux États-Unis. Les multinationales américaines n'investissent pas au Canada pour contourner les barrières tarifaires ou non tarifaires. Ces décisions répondraient essentiellement aux besoins d'accès à de nouveaux marchés et de diversification. Toutefois, les investisseurs américains seraient, d'une part, largement influencés par la réglementation gouvernementale et, d'autre part, seraient sensibles

8. Cette enquête n'a cependant été réalisée qu'auprès d'un nombre limité de firmes multinationales américaines implantées au Canada, dont notamment les plus importantes telles Ford of Canada, Imperial Oil, IBM Canada, Amoco Canada, Mobil Oil Canada, Cargill, Canadian General Electric, Dow Chemical Canada, Dupont Canada and Procter and Gamble.

9. Mentionnons que certe enquête s'adressait à d'importantes multinationales américaines ayant des filiales au Canada. Elle reflète le point de vue des maisons mères, point de vue qui peut différer des dirigeants des fliales concernant l'impact éventuel d'un accord de libre-échange. 
aux coûts de la main-d'œuvre. En ce sens, il est clair que le libreéchange exercerait des pressions importantes visant à l'harmonisation des politiques (fiscalité, réglementation, environnement, éducation) ce qui se traduirait vraisemblablement par une diminution des coûts variables imposées par les divers programmes gouvernementaux. L'appui d'une partie de la fraction compradore de la bourgeoisie trouve ses assises dans ces dernières considérations.

La politique commerciale libre-échangiste semble donc profitable aux grandes entreprises qui exportent déjà une partie de leur production ou qui songent à s'implanter à l'étranger. De la même manière, elle est favorisée par les intérêts étrangers qui y voient la possibilité de réduire l'intervention étatique.

Cependant, d'autres études ont démontré des clivages importants entre les firmes étrangères implantées au Canada. Une enquête réalisée par l'Association des manufacturiers canadiens, s'adressant non pas aux sociétés mères mais plutôt à leurs filiales canadiennes, indiquait que plus du tiers estimaient qu'elles réduiraient leurs activités suite à l'adoption d'une politique de libreéchange (Canada, 1980, fascicule 14, 50). Notons que cette enquête ne s'adressait pas uniquement aux grandes multinationales américaines: environ $85 \%$ de l'échantillonnage était constitué de petites et moyennes entreprises (i.e. avec un actif de 50 millions de dollars ou moins). Qui plus est, les entreprises sous contrôle étranger semblent même davantage vulnérables au libre-échange que les entreprises sous contrôle canadien. Les filiales qui ont été implantées pour répondre aux besoins du marché canadien manifestent beaucoup de crainte alors que celles qui exportent déjà une partie de leur production aux États-Unis ou vers d'autres marchés, parce que disposant bien souvent de mandats continentaux ou mondiaux de production, sont quant à elles peu inquiètes des retombées d'une plus grande intégration puisqu'elles sont structurées pour faire face à la concurrence continentale et internationale (Canada, 1980, fascicule 14, 51). 
La prise en compte des intérêts des grandes entreprises œuvrant au Canada laisse déjà percevoir la ligne de démarcation entre les différents éléments qui composent la bourgeoisie canadienne. Ainsi, il semble se dégager que les sociétés qui sont peu orientées vers les exportations sont celles qui sont le plus vulnérables à une possible libéralisation commerciale. Près de $40 \%$ des firmes qui n'exportent pas ont estimé que leur chiffre d'affaires diminuerait. L'inverse est évidemment vrai: plus les exportations sont importantes, moins les sociétés sont vulnérables dans la mesure où elles sont déjà en position pour soutenir la concurrence.

L'ensemble du grand capital, peu importe sa structure de propriété, ne tirerait donc pas nécessairement avantage d'une grande intégration continentale via une libéralisation des échanges (Canada, 1980, fascicule 14, 52). Il y a presque autant de très grandes entreprises qui verraient leurs activités diminuées $(30,8 \%)$ qu'il y en a qui les augmenteraient $(28,8 \%)$. Ainsi, on ne peut pas conclure que ce sont nécessairement les plus grandes sociétés qui s'en tireraient le mieux. Certaines d'entre elles se verraient obliger de restructurer leurs activités sans pour autant les élargir.

Alors que la fraction non monopoliste de la bourgeoisie devrait théoriquement s'opposer à l'accentuation du lien de dépendance, elle semble au contraire appuyer une telle approche. Un sondage mené à la fin juin 1986 par la Fédération canadienne de l'entreprise indépendante, qui compte 76,000 membres représentant près de $10 \%$ de toutes les PME canadiennes, indiquair que 35,4\% d'entre elles croyaient qu'un accord canado-américain les avantagerait, pour $32,5 \%$ l'impact serait nul et seulement $13,9 \%$ anticipaient un impact négatif ${ }^{10}$.

Par ailleurs, un autre sondage conduit par le Conseil canadien des petites entreprises, organisme qui compte 6,000 membres

10. Ces données ont été rapportées dans Le Devoir économique, III, I, (février 1987), 24 . 
provenant à $85 \%$ d'entreprises de moins de 20 employés, indiquait que $66 \%$ des répondants étaient favorables à un accord commercial global contre seulement $20 \%$ qui s'y opposaient. Ainsi, selon le $\mathrm{CCPE}$, «il ne reste à peu près rien de la polarisation régionale sectorielle qui a caractérisée certains débats antérieures sur les changements de structure économique» (Canada, 1985, fascicule $4,45)$. Toutefois, cette intégration ne devrait pas s'établir selon les mêmes termes pour tous les secteurs. Outre une période d'ajustement d'environ cinq ans, le gouvernement central doit prévoir des mesures d'exception ou accorder des garanties à l'égard d'industries spécifiques, notamment dans le secteur de production des biens primaires comme les pêcheries, l'agriculture et la foresterie.

Bien entendu, nous devons interpréter avec circonspection les résultats de ces sondages reflétant les positions des entreprises de petite et de moyenne envergure. Les membres de la FCEI ne représentent après tout que dix pour-cent des PME au Canada. Cet accord de la fraction non monopoliste de la bourgeoisie peut s'expliquer par le fait que ce sont les barrières non tarifaires qui constituent souvent les obstacles les plus importants à l'accès au marché américain: mentionnons notamment les conditions rigides et les règlements ayant trait au financement des transactions internationales, les délais aux douanes, la classification et l'évaluation «malveillantes» des biens importés, les mesures visant à contrebalancer le soutien des prix par le gouvernement, les subventions, les politiques d'achats gouvernementales, l'inefficacité du service postal, etc. (Canada, 1985, fascicule 4, 45-46). Par ailleurs, la plupart des petites entreprises n'exportent pas directement leur production. Celle-ci est cependant consommée par des entreprises plus importantes qui sont engagées dans des activités exportatrices. Il peut donc y avoir adéquation entre les intérêts des entreprises œuvrant dans les mêmes branches industrielles. Comme le soulignait le président de la FCEI, «établir des entreprises en participation avec des intérêts étrangers est la voie à explorer et que la PME 
est le meilleur outil à cause de sa souplesse" ". Rappelons que les PME ne doivent généralement pas leur survie à l'existence de subventions, ces dernières étant absorbées par les grandes entreprises (le CCPE cite le cas de la Société pour l'expansion des exportations où 10 entreprises accaparaient $50 \%$ des subventions). L'appui de la petite bourgeoisie d'affaire repose sur des considérations économiques, mais aussi idéologiques. Cette fraction de la classe dominante partage le crédo néo-libéral qui se cache derrière l'orientation libre-échangiste.

Il ressort de toutes ces observations une remise en question du consensus qui semble se dégager des prises de position de principales associations patronales.

\section{Conclusion}

Le mode d'appréhension fondé sur les fractions de classes, i.e. fondé sur un partage structurel des champs d'accumulation entre fractions monopoliste ou non-monopoliste / compradore ou autochtone, est inadéquat pour rendre compte de l'alliance des différentes composantes de la classe économiquement dominante à l'égard du processus de continentalisation économique. Nous pouvons tout de même avancer une autre interprétation tenant davantage compte du processus d'accumulation du capital caractérisant les différents secteurs de l'économie canadienne. Ainsi, règle générale, le soutien à une politique de libéralisation commerciale canado-américaine est fonction de la nature des marchés. Les firmes déjà orientées vers le marché continental font la promotion de la libéralisation du commerce bilatéral puisqu'une telle politique leur permettrait d'accroître le niveau de leurs exportations. Leur argumentation est fondée sur les bienfaits économiques éventuels,

11. "Le libre-échange: intervenants et enjeux", La revue de CMA. (mai-juin 1986), 22. 
à long terme, d'un élargissement des marchés, mais aussi sur des considérations conjoncturelles liées à la montée du protectionnisme américain. Un tel parti-pris ne signifie pas qu'elles renoncent automatiquement aux interventions de l'État qui leur sont favorables, mais ces politiques doivent être assujetties aux conditions du commerce continental.

Par ailleurs, les firmes dont le principal marché est limité au Canada sont moins bien disposées à l'accroissement des relations économiques bilatérales; elles craignent que la production américaine n'envahisse le marché canadien si toutes les restrictions commerciales sont levées. Les producteurs qui écoulent leurs produits sur le marché canadien ne pourraient faire face, dans l'immédiat, aux producteurs américains qui disposent d'une structure industrielle souvent mieux intégrée et davantage rationalisée. Ce point de vue n'est pas avancé que par les éléments autochtones de la bourgeoisie, mais aussi par plusieurs filiales de sociétés américaines qui craignent une relocalisation de leur production aux États-Unis. La fraction compradore de la bourgeoisie affiche une position tenant compte aussi de la nature des marchés: les secteurs économiques et les branches manufacturières qui sont dépendants du marché national réclament donc la poursuite des politiques qui sans être nommément protectionnistes, assurent un soutien indispensable à leur survie et à leur développement. Les perspectives de croissance pour les entreprises détenues par des capitaux canadiens seraient meilleures si ces firmes étaient plus importantes, plus sophistiquées («high tech») et davantage tournées vers l'exportation (Litvak et Warner, 1987, 14-19).

Ce ne sont donc pas essentiellement la structure de propriété ou même la taille des entreprises qui sont globalement déterminantes dans le fait d'appuyer ou non une plus grande ouverture du marché canadien à la concurrence américaine. Nous constatons même que plusieurs entreprises de moindre importance comptent tirer un plus grand avantage de la création d'une zone de libre-échange 
que les entreprises de plus grande envergure. Toutefois, il semble que l'approfondissement des rapports bilatéraux nécessiterait des réajustements pour tous les secteurs et branches industrielles, quelle que soit l'importance des sociétés.

Les fractions financières et commerciales de la bourgeoisie continuent à privilégier la croissance des échanges bilatéraux dans la mesure où ceux-ci permettent d'augmenter la circulation du capital. La provenance des investissements est quant à elle secondaire (Langille, 1987).

En somme, la stratégie d'intégration continentale est perçue positivement tant de la part d'entreprises étrangères que de firmes canadiennes de grande ou de moyenne envergure. L'accès élargi au marché continental encouragerait le processus de spécialisation et de concentration industrielle au détriment des firmes n'ayant traditionnellement desservi que le marché intérieur. Nous assistons donc à une nouvelle forme de développement dépendant associé où d'importants éléments de la bourgeoisie compradore, dans des secteurs en déclin, se voient obligés de se retrancher pour faire face aux problèmes de la concurrence internationale. Ces éléments doivent de plus se soumettre aux éléments de la bourgeoisie qui cherchent à accroître leur champ d'action sur le marché nordaméricain. L'alliance de classe qui se dégage regroupe les entreprises qui exportent ou qui dépendent du marché continental, entreprises déjà en mesure de concurrencer sur les marchés nord-américains et international. En ce sens, il n'existe pas d'antinomie entre l'approfondissement de l'intégration continentale et le processus de maturation de la bourgeoisie canadienne, mais plutôt un rapport de complémentarité qui se situe dans la mouvance d'une nouvelle forme de développement dépendant. 


\section{BIBLIOGRAPHIE}

Association des manufacturiers canadiens, (1984), Un avenir qui promet, (septembre), 135. Association des manufacturiers canadiens, (1987), Accord de libre-échange Canada-É.U., Base fondamentale de l'avenir. Document stratégique de l'Association des manufacturiers canadiens, (18 novembre), 19.

Brunelle, Dorval, Deblock, Christian, (1987), «Défenseurs et adversaires du libre-échange avec les U.S.A.: Études des positions des parties au Canada», in P. J. Hamel, Un marché, deux sociétés? 2"me partie. Libre-échange; aspects socio-économiques, (Montréal, ACFAS), 25-41.

Cameron, Duncan, (1986), (ed.), The Free Trade Papers, (Toronto, James Lorimer), 227.

Canada, (1980), Délibération du Comité sénatorial permanent des Affaires étrangères, $1^{\text {ère }}$ session, $32^{e}$ législature.

Canada, (1985), Procès-verbaux et témoignages du Comité mixte spécial du Sénat et de la Chambre des communes sur les Relations extérieures du Canada, première session de la trentetroisième législature, 1984-1985.

Canadian Export Association, (1983), Mémoire soumis à la Commission royale d'enquête sur l'union économique et les perspectives de développement du Canada (5 octobre).

Canadian Manufacturers' Association, (1983), Future Making: The Era of Human Ressources. Mémoire soumis à la Commission royale sur l'union économique et les perspectives de développement du Canada, (6 septembre).

Carroll, William K., (1986), Corporate Power and Canadian Capitalism, (Vancouver, University of British Columbia Press), 284.

Clement, Wallace, (1975), The Canadian Corporate Elite, (Toronto, McClelland and Stewart), 479.

Clement, Wallace, (1977A), Continental Corporate Power, (Toronto, McClelland and Srewart), 408.

Clement, Wallace, (1977B), "The Canadian Bourgeoisie - Merely Comprador», in C. Heron et J. Saul (ed.), Imperialism. Nationalism and Canada. (Toronto/Kitchener, New Hogtown Press/Between the Lines), 71-84.

Clement, Wallace, (1978), "Canada and Multinational Corporations: An Overview", in D. Glenday, H. Guindon et A. Turowetz (eds), Modernisation and the Canadian State, (Toronto, Macmillan), 18-35.

Clement, Wallace, (1983), Class, Power and Property: Essays on Canadian Society, (Toronto, Methuen).

Crookell, Harold, (1984), "Specialization and International Competitiveness", in Business Quaterly, 49, (Fali), 26-31.

Drache, Daniel, Kroker, Arthur, (1983), "The Labyrinth of Dependency", in Revue canadienne de théorie politique et sociale, VII, 3, (automne), 5-24.

Drache, Daniel, Wallace Clement, (1985), The New Practical Guide to Canadian Political Economy, (Toronto, James Lorimer), 243.

Éthier, Diane, (1983), «Le Canada et la nouvelle division internationale du travail: une problématique à développer", in Revue canadienne d'études du développement. IV, 2 , 346-358.

Éthier, Diane, (1985), «L'économie politique canadienne et la nouvelle division internationale du travail», in Duncan Cameron et François Houle (eds), Le Canada et la nouvelle 
division internationale du travail. (Ottawa, Éditions de l'Université d'Ottawa), 5978.

Finlayson, J. A., (1985), "Canadian Business and Free Trade», in International Perspectives, (March/April).

Fournier, Pierre (dir.), (1978), Le Capitalisme an Québec, Montréal, St-Martin.

Glenday, Daniel, (1983), "The 'dependencia' school in Canada: an examination and evaluation", in Revue canadienne de sociologie et d'antbropologie, XX, 3, 346-358.

Houle, François, (1987), "L'Érat canadien et le capitalisme mondial: stratégies d'insertion", Revue canadienne de science politique, XX, 3, (septembre), 467-500.

Hutcheson, John, (1973), "The Capitalist State in Canada", in R. Laxer, (ed), Canada Ltd. (Toronto, McCelland and Stewart), 153-177.

International Business Council of Canada, (1983), Mémoire soumis à la Commission royale sur l'union économique et les perspectives de développement du Canada, (28 novembre), 8.

Kierans, Eric, (1987), "Corporations Over All», in Policy Options Politiques, 8, (June) 69.

Langille, David, (1987), "The Business Council on National Issues and the Canadian State», in Studies in Political Economy, 24, (Autumn), 41-85.

Langille, David, (1988), "The BCNI Calls the Shots», in The Facts, X, 2, (Spring), 102 107.

Laxer, Robert, (1973), (ed), Canada Ltd. (Toronto, McCelland and Stewart), 271.

Layton, Jack, (1976), "Nationalism and the Canadian Bourgeoisie: Contradictions of Dependence", in Revue canadienne des études sur le nationalisme, III, 2, (printemps), 146-171.

Levitt, Kari, (1973), La capitulation tranquille, (Montréal, L'étincelle), 222.

Litvak, I. A., (1986), "Freer Trade With the U.S. : The Conflicting Views of Canadian Business", in Business Quaterly, 51, 1, (Spring), 22-32.

Litvak, I. A., Warner, T. N., (1987), "Multinationals, Advanced Manufacturing Technologies, and Canadian Public Policy", in Business Quaterly, LII, 1, (Summer), 14-19.

Lumsden, Ian, (1979), "Thoughts on Canadian Dependency", in Daniel Drache (ed), Debates and Controversies from this Magasine, (Toronto, McClelland and Stewart), 12-28.

Ministère des affaires extérieures, (1986?), Effect on Enhenced Trade on Investment: Survey Evidence, Confidential document, 16.

Niosi, Jorge, (1979), «Le contrôle des filiales érrangères au Canada: nouvelles perspectives sur la bourgeoisie compradore», in Sociologie et Sociétés, XI, 2, (octobre), 97-123.

Niosi, Jorge, (1980), La bourgeoisie canadienne, (Montréal, Boréal Express), 243.

Niosi, Jorge, (1982), Les multinationales canadiennes, (Montréal, Boréal Express), 222.

Niosi, Jorge, (1983), "The Canadian Bourgeoisie: Towards a Synthetical Approach", in Revue Canadienne de théorie politique et sociale, VII, 3, (Automne), 128-149.

Panitch, Leo, (1977), "The Role and Nature of the Canadian State», in C. Heron et J. Saul (ed), Imperialism. Nationalism and Canada. (Toronto/Kitchener, New Hogtown Press/Between the Lines), 85-107.

Panitch, Leo, (1981), "Dependency and Class in Canadian Political Economy», in Studies in Political Economy, 6, (Autumn), 7-33.

Resnick, Philip, (1982), "The Maturing of Canadian Capitalism», in Our Generation. $\mathrm{XV}, 3$, (Fall), 11-24. 
Resnick, Philip, (1988), From Semi-peripbery to Perimeter of the Core: Canada's Place in the Capitalist World Economy, (à paraitre).

Rugman, Alan M., (1987), "Living Wiht Free Trade: How Multinationals Will Adjust to Trade Liberalization", in Business Quaterly, LII, 2, (Fall), 85-90.

Schmidt, Ray, (1981), "Canadian Regionalism in Continental Perspective», Studies in Political Economy, 6, (Autumn), 65-92.

Stevenson, Garth, (1980), "Canadian Regionalism in Continental Perspective", Journal of Canadian Studies, 15, (Summer), 16-28.

Stevenson, Garth, (1982), Unfulfilled Union: Canadian Federalism and National Unity. (Toronto, Gage), 257. 